\title{
Scaling Properties of Flexible Membranes from Atomistic Simulations: Application to Graphene
}

\author{
J. H. Los ${ }^{1}$ M. I. Katsnelson, ${ }^{1}$ O. V. Yazyev ${ }^{2}{ }^{3}$ K. V. Zakharchenko, ${ }^{1}$ and A. Fasolino ${ }^{1}$ \\ ${ }^{1}$ Institute for Molecules and Materials, Radboud University Nijmegen, \\ Heyendaalseweg 135, 6525 AJ Nijmegen, The Netherlands \\ ${ }^{2}$ Ecole Polytechnique Fédérale de Lausanne (EPFL), \\ Institute of Theoretical Physics, CH-1015 Lausanne, Switzerland \\ ${ }^{3}$ Institut Romand de Recherche Numérique en Physique des Matériaux (IRRMA), CH-1015 Lausanne, Switzerland
}

(Dated: March 16, 2022)

\begin{abstract}
Structure and thermodynamics of crystalline membranes are characterized by the long wavelength behavior of the normal-normal correlation function $G(q)$. We calculate $G(q)$ by Monte Carlo and Molecular Dynamics simulations for a quasi-harmonic model potential and for a realistic potential for graphene. To access the long wavelength limit for finite-size systems (up to 40000 atoms) we introduce a Monte Carlo sampling based on collective atomic moves (wave moves). We find a power-law behaviour $G(q) \propto q^{-2+\eta}$ with the same exponent $\eta \approx 0.85$ for both potentials. This finding supports, from the microscopic side, the adequacy of the scaling theory of membranes in the continuum medium approach, even for an extremely rigid material like graphene.
\end{abstract}

PACS numbers: 63.20.Ry, 68.60.Dv, 81.05.Uw, 05.10.Ln

Collective phenomena involving infinitely many degrees of freedom are often characterized by scaling laws with power-law behavior of correlation functions. In three dimensional systems, this behavior occurs only at critical points [1, 2, 3]. In two dimensions (2D) the situation is different, and a whole temperature interval with "almost broken symmetry" and power-law decay of correlation functions frequently appears, the KosterlitzThouless (KT) transition in 2D superfluids and superconductors [4] being a prototype example. Existence of real long range order, where correlation functions remain non-zero in the limit of infinite distance, is forbidden in such cases by the Mermin-Wagner theorem [5] due to the divergence of the contribution of soft modes to relevant thermodynamic properties. The theory of flexible membranes [6] embedded in higher dimensions is an important part of the statistical mechanics of $2 \mathrm{D}$ systems. Here, we investigate the scaling behavior of crystalline flexible membranes by means of atomistic simulations, using graphene [7, 8, [9], the simplest known membrane, as an example.

In the flat phase, the membrane in-plane and out-ofplane displacements are parametrized by a $D$-component 'stretching' phonon field $u_{\alpha}(\mathbf{x}), \alpha=1 \ldots D$, and by a $d_{c}=d-D$ component out-of-plane height fluctuation $h(\mathbf{x})$, where $d$ is the space dimension and $D$ is the membrane dimension. Softening of bending modes makes this situation very similar to the KT model. A minimal phenomenological model for membranes is just the elasticity theory described by the Hamiltonian [6, 10]:

$$
H=\frac{1}{2} \int d^{D} x\left(\kappa\left(\nabla^{2} h\right)^{2}+\mu u_{\alpha \beta}^{2}+\frac{\lambda}{2} u_{\alpha \alpha}^{2}\right)
$$

where $\kappa, \mu$ and $\lambda$ are bending rigidity, shear modulus and Lamé coefficient and

$$
u_{\alpha \beta}=\frac{1}{2}\left(\partial_{\alpha} u_{\beta}+\partial_{\beta} u_{\alpha}+\partial_{\alpha} h \partial_{\beta} h\right)
$$

is the strain tensor. In harmonic approximation, by neglecting the last, non-linear, term in Eq. (2), the bending $(h)$ and stretching (u) modes are decoupled.

The Hamiltonian (1) is quadratic in the phonon degrees of freedom $\mathbf{u}$ which can be eliminated by Gaussian integration [6, 10]. In this way, the Hamiltonian can be rewritten only in terms of the Fourier components of the height $h$ as the sum of a harmonic bending energy, quadratic in $h$, and an anharmonic energy, quartic in $h$, that results from the coupling of bending and stretching modes [10]. If one neglects the latter term, the membrane becomes crumpled at any finite temperature with, for $D=2$, the mean square height fluctuations $\left\langle h^{2}\right\rangle \sim L^{2}$ and normal-normal correlation functions that diverge logarithmically at large distances. Nelson and Peliti [10] suggested that the above anharmonic term stabilizes the flat phase at least at temperatures much smaller than $\kappa$. This flat phase is described by an effective bending rigidity $\kappa(q) \sim q^{-\eta}$ and effective elastic moduli with power-law dependencies on $q$ that partially suppress long wavelength bending fluctuations. As a result, the normal-normal correlation function remains finite, although $\left\langle h^{2}\right\rangle$ still diverges as $\left\langle h^{2}\right\rangle \sim L^{2 \zeta}$ with $\zeta=1-\eta / 2[\underline{6}]$. Thus, the flat phase is not truly flat, but still exhibits rather strong corrugation. The model (11), which is called the model of phantom membranes, has a transition to a crumpled phase at temperature of the order of $\kappa$. The long wavelength limit was solved within the Self Consistent Screening Approximation in Ref. 11 yielding $\eta=0.821$. The discretized version of this model was investigated by Bowick et al. by means of Monte Carlo simulations giving $\eta \approx 0.72[12]$. The term 'phantom' means that the model does not include self-avoidance, the natural condition of true physical systems. It is assumed that self-avoidance removes the phase transition to the high temperature crumpled phase while the scaling properties of the 'flat' phase remain the same 
as in phantom membranes. However, any kind of accurate statement about the model can be justified only in the limit $d_{c} \rightarrow \infty$ and, strictly speaking, nothing can be said rigorously for the real case of $d=3, D=2$ and $d_{c}=1$.

To characterize the long wavelength limit of the height fluctuations we compare the results of atomistic simulations to the predictions of this theory for the normalnormal correlation functions $G(q)=\left\langle\left|\mathbf{n}_{q}\right|^{2}\right\rangle$. Starting from Eq. (1) an expression for $G(q)$ has been given from general scaling considerations $[6,10,15]$ in the form of an effective Dyson equation

$$
G_{a}^{-1}(q)=G_{0}^{-1}(q)+\Sigma(q)
$$

where $G_{0}$ is the value derived in harmonic approximation

$$
G_{0}(q)=\frac{T N}{\kappa S_{0} q^{2}}
$$

and the self energy is

$$
\Sigma(q)=\frac{A S_{0}}{N} q^{2}\left(\frac{q_{0}}{q}\right)^{\eta}
$$

with $N$ the number of atoms, $S_{0}=L_{x} L_{y} / N$ the area per atom, $T$ the temperature in units of energy, $q_{0}=$ $2 \pi \sqrt{B / \kappa}, B$ the two-dimensional bulk modulus [13] and $A$ an unknown numerical factor.

Until recently, this phenomenological continuum model was the only way to describe the statistical mechanics of membranes since all known real membranes [6] were too complicated for atomistic models. The situation has been changed drastically by the discovery of graphene 7 ] which is the first example of a truly two-dimensional system (just one atom thick) and, thus, a prototype crystalline membrane [8, 9]. The experimental observation of ripples in freely hanged graphene 14 stimulated a large theoretical activity [15, 16, 17, 18, 19, 20, 21]. In particular, using the accurate bond order potential for carbon LCBOPII 22], we were able to simulate structural and thermodynamical properties of graphene at finite temperatures [15, 21] by straightforward Monte Carlo (MC) simulations. The simulations confirmed the existence of thermally induced intrinsic ripples at finite temperatures resulting in strong anharmonic effects. However, we found that the normal-normal correlation function could not be described by Eq. (3) over the whole range of $q$ [15]. In fact, $G(q)$ followed the power law resulting from the harmonic approximation (phonon picture, $\eta=0$ ) at large enough $q$, but, after bending, at smaller $q$ 's we found a drop of the correlation functions not compatible with a power law. Our conjecture at that time was that the extreme rigidity of graphene could be the reason why it could not be described by the phenomenological theory of membranes in a continuum medium approach [10]. However, we felt that this point deserved further investigation. Here, we focus on the low- $q$ region in order to establish firmly whether a scaling law exists and, if so, to determine the scaling exponent. To this purpose, we simulate large systems, introduce new MC moves for phase space sampling and examine more than one model of the interatomic forces, including a simple quasi-harmonic $(\mathrm{QH})$ model that yields a not too rigid membrane and the extremely rigid case of graphene which is well described by LCBOPII. In addition, for the QH model we verified ergodicity of our MC simulations by comparing with Molecular Dynamics (MD) results.

We begin by considering a relatively simple $\mathrm{QH}$ model with energy given by:

$$
U=\frac{1}{2} \sum_{i} \sum_{j \neq i}\left(K_{r}\left(r_{i j}-r_{e q}\right)^{2}+K_{\theta} \sum_{k \neq i, j}\left(y_{i j k}-y_{e q}\right)^{2}\right)
$$

where the summations over $j$ and $k$ are over the nearestneighbors of atom $i, y_{i j k}=\cos \theta_{i j k}$ and $y_{e q}=\cos \theta_{e q}$, with $r_{e q}=1.42 \AA$ and $\theta_{e q}=2 \pi / 3$ the ground state equilibrium nearest neighbor distance and bond angle in graphene. The stretching and the bending force constants, $K_{r}=22 \mathrm{eV} \AA^{-2}$ and $K_{\theta}=4 \mathrm{eV}$, respectively, were chosen to yield elastic moduli for isotropic and uniaxial compressions equal to those for the LCBOPII [21].

In Fig. 1 we show the function $G(q) / N$ (dotted line) calculated by extensive standard Monte Carlo simulations in the canonical ensemble at $300 \mathrm{~K}$ for a system with $N=37888, L_{x}=314.82 \AA$ and $L_{y}=315.24 \AA$ and periodic boundary conditions in the $x y$-plane. Starting from the Bragg peak at $q=4 \pi /\left(3 r_{e q}\right)=2.94 \AA^{-1}$ and going towards lower $q$ we find, first, the power law $\eta=0$ due to the harmonic contribution, then, a smaller slope followed by a drop at the smallest $q<0.08 \AA^{-1}$ which corresponds to a wavelength of about $75 \AA$. This drop is similar to the one mentioned above and found previously in Ref. 15 with the LCBOPII for graphene. These results are obtained by averaging over many configurations in the canonical ensemble obtained by the ordinary MC procedure which is based on random displacements of randomly chosen individual atoms and volume (area) fluctuations with a Metropolis acceptance rule. By using Eq. (4) we find that the bending constant for the $\mathrm{QH}$ potential is $\kappa=0.4 \mathrm{eV}$, much softer than the $1.1 \mathrm{eV}$ appropriate for graphene [15], due to neglected interactions beyond first neighbors. The observation that also the simple QH model shows a suppression of long wavelength excitations made us think of the possibility that standard $\mathrm{MC}$ is not an efficient sampling technique in this case. To resolve this issue we (i) extended our MC phase space sampling with a new type of collective trial events that we call 'wave moves' described below, and (ii) performed MD simulations for the QH model [23], allowing a direct comparison with the MC results, with and without wave moves. The equivalence of time averages in MD simulations with ensemble averages in MC simulations guarantees that the system is in thermodynamical equilibrium (ergodic).

In Fig. 1 we compare the results of standard MC with the results obtained by MD and by $\mathrm{MC}$ with the addition of wave moves. The MD results coincide with the stan- 


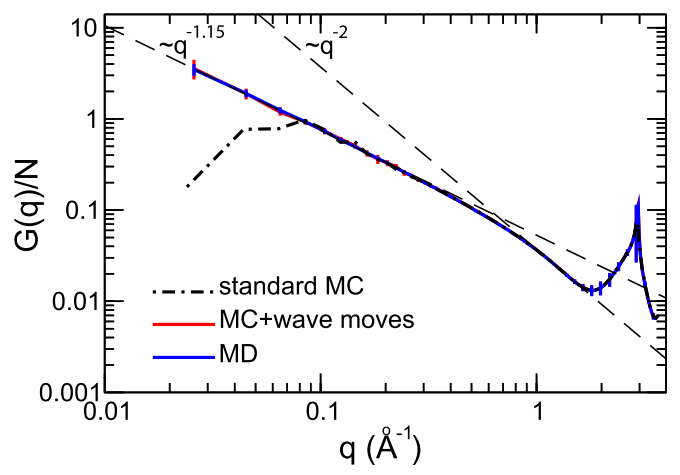

FIG. 1: (color online) Normal-normal correlation functions $G(q) / N$ calculated for a graphene system with $N=37888$ by ordinary MC simulations (red-dashed line), MD simulations and MC simulations with wave moves with the $\mathrm{QH}$ potential. The dashed lines show the asymptotic harmonic behavior with power laws $q^{-2}$ for large $q$ and the long-wavelength limit $q^{-(2-\eta)}$ with $\eta=0.85$.

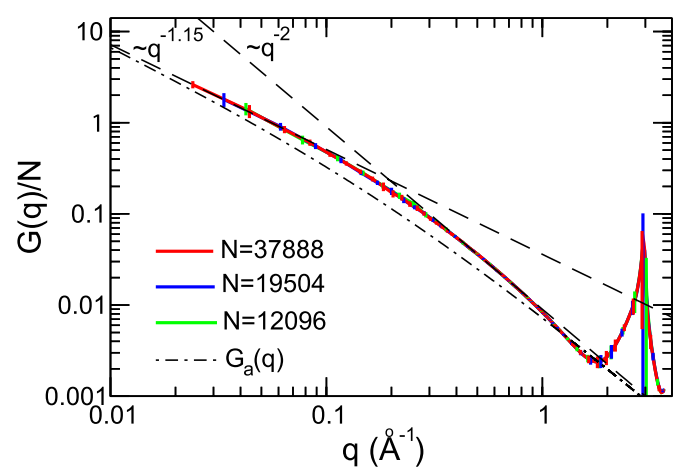

FIG. 2: (color online) Normal-normal correlation functions $G(q) / N$ calculated for three systems with $N=12096\left(L_{x}=\right.$ $\left.177.08 \AA, L_{y}=178.92 \AA\right), N=19504\left(L_{x}=226.27 \AA, L_{y}=\right.$ $225.78 \AA), N=37888\left(L_{x}=314.82 \AA, L_{y}=315.24 \AA\right)$ by MC simulations with wave moves with LCBOPII. The dashed lines show the asymptotic harmonic behavior with power laws $q^{-2}$ for large $q$ and the long-wavelength limit $q^{-(2-\eta)}$ with $\eta=0.85$. The dashed-dotted line is $G_{a}$ of Eq. (3) with the coefficients fixed by the asymptotic behavior. One can see that the crossover is much sharper in the simulations.

dard MC in the range where the latter is described by a power law, but does not show the drop at small $q$ and keeps the same slope till the smallest possible $q$ allowed by our finite size system. The results of MC simulations with wave moves coincide for all $q$ 's with those obtained by $\mathrm{MD}$, implying that the system is in thermodynamic equilibrium. Both curves display a power-law behavior for the whole range of $q$ in the long wavelength limit. A best fit of the data yields an exponent $\eta=0.85$.

A wave move consists of a transversal, wavelike displacement of all atoms in the $z$-direction, perpendicular to the graphene plane. For a given wavevector $\mathbf{q}$ there are two possible, linearly independent wave excitations, yielding $z$-coordinate displacements for all atoms $i$

$$
\begin{aligned}
\Delta \mathbf{z}_{i} & =(0.5-R) A_{S, q} \cos \left(\mathbf{q} \mathbf{r}_{i}\right) \quad \text { and } \\
\Delta \mathbf{z}_{i} & =(0.5-R) A_{S, q} \sin \left(\mathbf{q} \mathbf{r}_{i}\right)
\end{aligned}
$$

where $\mathbf{r}_{i}$ is the 3D position of atom $i$ and $R$ is a random number between 0 and 1 . The amplitude $A_{S, q}$ is chosen such that the acceptance rate for such a wave move is between 0.4 and 0.5 . The appropriate value of $A_{S, q}$ depends on the size of the 2D box $S=L_{x} L_{y}$ and on the wavevector $\mathbf{q}$ (see below).

Due to the periodic boundary conditions in the $x$ - and $y$-directions the candidate wavevectors for wave moves can be restricted to a set on a $2 \mathrm{D}$ grid:

$$
\mathbf{q}=\left(m_{x} \frac{2 \pi}{L_{x}}, m_{y} \frac{2 \pi}{L_{y}}, 0\right)
$$

with integer $m_{x}$ and $m_{y}$. This set was further bounded by applying only wave moves of long wavelengths since short wavelengths are already efficiently sampled by the individual atom displacement trials. Hence, we consider a finite set of $\left(m_{x}, m_{y}\right)$-pairs corresponding to $q$-vectors within a circular region with radius $q_{\max }$ around $\mathbf{q}=0$. This set was kept constant during the entire simulation. We choose $q_{\max }$ equal to the $q$-value below which $G(q)$ starts to bend down in standard MC simulation. More precisely, we took $q_{\max } \simeq 0.16$, corresponding to a minimal wavelength of $40 \AA$. Since transversal phonon modes have quadratic dispersion $\omega(q) \sim q^{2}$, the energy change associated with a wave move behaves as $\Delta E_{w m} \sim A_{S, q}^{2} q^{2}$. Therefore, we took $A_{S, q}=A_{S} / q$ to obtain similar acceptance rates for each of the allowed $q$-vectors, as was indeed confirmed by our simulations. This choice leaves one adjustable parameter, $A_{S}$. For different system sizes, the appropriate $A_{S}$ roughly scales as $A / S$, but a correction is required to fine-tune the acceptance rate. On average, a wave move was attempted every MC step by choosing randomly one of the $2 N_{q}$ possible waves. Here, $N_{q}$ is the number of allowed $q$-vectors (or $\left(m_{x}, m_{y}\right)$-pairs) and the factor 2 comes from the fact that each wavevector yields two possible waves: a sine and a cosine wave. Another random number $R \in(0,1)$ was then pulled to fix the amplitude $(0.5-R) A_{S} / q$. Following the Metropolis procedure, a wave move is always accepted if the energy change $\Delta E_{w m}$ is negative, whereas for $\Delta E_{w m}>0$ it is accepted with probability $P=\exp \left(-\beta \Delta E_{w m}\right)$, requiring another random number $R^{\prime} \in(0,1)$ to decide for acceptance when $R^{\prime} \leq P$ or rejection when $R^{\prime}>P$.

MD simulations are much more demanding than MC simulations and are not within reach for the rather complex LCBOPII potential for the present system size. The previous results with the $\mathrm{QH}$ harmonic potential, however, show that equilibrium can be reached using $\mathrm{MC}$ with wave moves. The correlation function $G(q)$ calculated by MC with wave moves for LCBOPII are shown in Fig. 2 for three system sizes. Again, we see the crossover from the harmonic behavior to a power law with $\eta=0.85$ up to the smallest wavevectors. The main difference with 

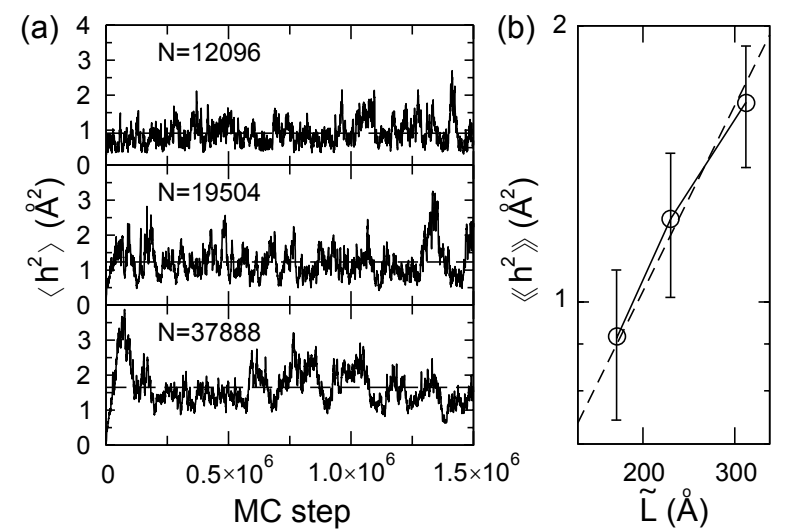

FIG. 3: (a) Quadratic out-of-plane displacement $\left\langle h^{2}\right\rangle$, averaged over all particles, as a function of the MC step for the same systems as in Fig. 2. The dashed horizontal lines denote $\left\langle\left\langle h^{2}\right\rangle\right\rangle$, the average $\left\langle h^{2}\right\rangle$ over all MC steps except the first $3 \times 10^{5}$ steps of equilibration. (b) $\left\langle\left\langle h^{2}\right\rangle\right\rangle$ as a function of the average linear system size $\tilde{L}=\sqrt{L_{x} L_{y}}$ compared to the scaling law $\left\langle\left\langle h^{2}\right\rangle\right\rangle=C L^{2-\eta}$ with $C=0.00232$ and $\eta=0.85$ (dashed line). Both axis are in logarithmic scale.

the results obtained with the $\mathrm{QH}$ potential is that, due to a higher bending rigidity, the crossover between the two power laws is shifted to lower $q$ values. Moreover, we note that for $q>1 \AA^{-1}$ there is a deviation from a power law behavior just before the Bragg peak.

Finally, in Fig. 3(a) we show the average out-of-plane displacement $\left\langle h^{2}\right\rangle$ corresponding to the simulations for
LCBOPII of Fig. 2 which shows large fluctuations. In Fig. 3(b) we plot the values of $\left\langle h^{2}\right\rangle$ averaged over all $\mathrm{MC}$ steps as a function of the system size in comparison with the expected scaling law $\exp (2-\eta)$. Although it would have been impossible to deduce the scaling exponent from the three points in Fig. 3(b) due to the large error originating from the large fluctuations, these results are certainly compatible with the scaling exponent $\eta$ found by a fit of $G(q)$. With $\left\langle h^{2}\right\rangle=1.65 \AA^{2}$ for $\tilde{L}=315$ $\AA$ and $\eta=0.85$ we estimate $\sqrt{\left\langle h^{2}\right\rangle} \approx 9 \AA$ for $L=1 \mu \mathrm{m}$, well in the range of measured values [14].

In summary, we have shown by atomistic simulations that, in thermodynamic equilibrium, crystalline membranes display a power-law scaling behavior of the normal-normal correlation function, in qualitative agreement with continuum medium theory. For different models of interactions with different rigidities, we found the same exponent of anomalous bending rigidity $\eta \approx 0.85$. We have demonstrated that the efficiency of MC simulations for this type of systems can be greatly improved by introducing collective wave moves. On the basis of our results, we conclude that despite its extreme rigidity, graphene behaves as a prototype membrane opening new ways to study the intriguing physics of membranes on a system with well known interatomic interactions.

This work is part of the research program of the 'Stichting voor Fundamenteel Onderzoek der Materie (FOM)', which is financially supported by the 'Nederlandse Organisatie voor Wetenschappelijk Onderzoek (NWO)'.
[1] K. G. Wilson and J. Kogut, Phys. Rep. 12, 75 (1974).

[2] J. J. Binney, N. J. Dowrick, A. J. Fisher, and M. E. J. Newman, The Theory of Critical Phenomena (Oxford University Press, Oxford, 1992).

[3] J. M. Yeomans, Statistical Mechanics of Phase Transitions (Oxford University Press, Oxford, 1992).

[4] J. M. Kosterlitz and D. J. Thouless, J. Phys. C 6, 1181 (1973).

[5] N. D. Mermin and H. Wagner, Phys. Rev. Lett. 17, 1133 (1966).

[6] Statistical Mechanics of Membranes and Surfaces, edited by D. R. Nelson, T. Piran, and S. Weinberg (World Scientific, Singapore, 2004).

[7] K. S. Novoselov, A. K. Geim, S. V. Morozov, D. Jiang, Y. Zhang, S. V. Dubonos, I. V. Grigorieva, and A. A. Firsov, Science 306, 666 (2004).

[8] A. K. Geim and K. S. Novoselov, Nat. Mater. 6, 183 (2007).

[9] M. I. Katsnelson, Mater. Today 10, 20 (2007).

[10] D. R. Nelson and L. Peliti, J. Phys. (Paris) 48, 1085 (1987).

[11] P. Le Doussal and L. Radzihovsky, Phys. Rev. Lett. 69, 1209 (1992).

[12] M. J. Bowick, S. M. Catterall, M. Falcioni, G. Thorleifsson, and K. N. Anagnostopoulos, J. Phys. I (Paris) 6, 1321 (1996).
[13] The $T=0$ value of $B$ for graphene according to LCBOPII has been incorrectly reported in Ref. 15. The correct value is $B=12.7 \mathrm{eV} / \AA^{2}$.

[14] J. C. Meyer, A. K. Geim, M. I. Katsnelson, K. S. Novoselov, T. J. Booth, and S. Roth, Nature 446, 60 (2007).

[15] A. Fasolino, J. H. Los, and M. I. Katsnelson, Nat. Mater. 6, 858 (2007).

[16] F. Guinea, M. I. Katsnelson, and M. A. H. Vozmediano, Phys. Rev. B 77, 075422 (2008).

[17] M. S. Foster and I.L. Aleiner, Phys. Rev. B 77, 195413 (2008).

[18] F. Guinea, B. Horovitz, and P. Le Doussal, Phys. Rev. B 77, 205421 (2008).

[19] T. O. Wehling, A. V. Balatsky, A. M. Tsvelick, M. I. Katsnelson, and A. I. Lichtenstein, Europhys. Lett. 84, 17003 (2008).

[20] E.-A. Kim and A. H. Castro Neto, Europhys. Lett. 84, 57007 (2008).

[21] K. V. Zakharchenko, M. I. Katsnelson, and A. Fasolino, Phys. Rev. Lett. 102, 046808 (2009).

[22] J. H. Los, L. M. Ghiringhelli, E. J. Meijer, and A. Fasolino, Phys. Rev. B 72, 214102 (2005).

[23] The MD simulations are obtained for a slightly different QH potential where the term $K_{\theta}\left(\cos \theta_{i j k}-\cos \theta_{e q}\right)^{2}$ is changed to $\tilde{K}_{\theta}\left(\theta_{i j k}-\theta_{e q}\right)^{2}$. By choosing $\tilde{K}_{\theta}=3 K_{\theta} / 4$ 
the two expressions coincide to the lowest order. 